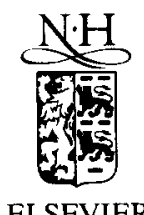

Applied Numerical Mathematics 18 (1995) 79-94

\title{
High-order multistep methods for boundary value problems
}

\author{
L. Brugnano, D. Trigiante * \\ Dipartimento di Energetica, Università di Firenze, Via C. Lombroso 6/17, 50134 Firenze, Italy
}

Received 19 September 1994; revised 15 November 1994; accepted 1 December 1994

\begin{abstract}
Linear multistep methods (LMMs) are extensively used for the numerical approximation of initial value problems (IVPs) for ODE. However, they are not commonly used to approximate continuous boundary value problems (BVPs), except in connection with the shooting method.

Recently, boundary value methods (BVMs) have been considered for the approximation of IVPs. We show that many of these methods can also be conveniently used to approximate with high accuracy continuous BVPs.
\end{abstract}

\section{Introduction}

In this paper we shall analyze the use of LMMs for the numerical approximation of continuous BVPs. The LMMs are not commonly used for approximating continuous BVPs, except in connection with the shooting method, where the continuous BVP is transformed into an IVP. This is in contrast with their extensive usage to approximate continuous IVPs. In fact, the most efficient codes for BVPs rely on one-step collocation schemes [4-6].

In the last few years LMMs have been used as boundary value methods (BVMs) for the approximation of continuous IVPs [1-3,7-11,13]. These methods replace the given continuous IVP by a suitable discrete BVP. In Section 2 the basic theoretical results on BVMs are briefly presented. In Section 3 we propose to use some BVMs to obtain high-order accuracy approximations of continuous boundary value problems. This is obtained by slightly modifying their use for IVPs. Thus, the same BVMs (and the same codes) can be used, with minor changes, to accurately solve both initial and boundary value problems. Their implementation is discussed in Section 4, where some numerical tests are also presented.

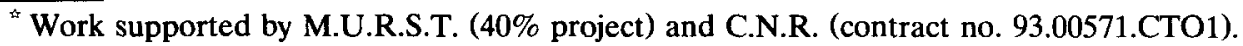

${ }^{*}$ Corresponding author. E-mail: mbxtrigiante@mail.cnuce.cnr.it.
} 


\section{Boundary value methods}

In this section we briefly recall the basic results on BVMs $[3,9,10]$. Let us first consider the IVP

$$
y^{\prime}=f(t, y), \quad t \in[a, b], \quad y(a)=y_{0},
$$

By considering the partition

$$
t_{i}=a+i h, \quad i=0, \ldots, N+k_{2}-1, \quad h=\frac{b-a}{N+k_{2}-1},
$$

one can consider the $k$-step LMM

$$
\sum_{i=0}^{k} \alpha_{i} y_{n+i}=h \sum_{i=0}^{k} \beta_{i} f_{n+i},
$$

to approximate the solution of problem (1) over the considered partition. As usual, $y_{n+i}$ and $f_{n+i}$ denote the approximations to $y\left(t_{n+i}\right)$ and $f\left(t_{n+i}, y\left(t_{n+i}\right)\right)$, respectively. It is known that the discrete problem (2) needs $k$ independent conditions to be imposed, in order to get the discrete solution. The most commonly used way of imposing such conditions is to fix the values of the discrete solution at the first $k$ grid points, that is one fixes the values $y_{0}, \ldots, y_{k-1}$. The continuous problem (1) provides only the first of these values (that is the initial condition $y_{0}$ ), while the remaining ones must be obtained by other means. In other words, the continuous IVP is approximated by means of a discrete IVP. The methods obtained in this way will be called initial value methods (IVMs). This approach is very simple, but suffers of heavy limitations, summarized by the two well-known Dahlquist barriers.

An alternative approach has been considered, where the $k$ conditions needed by the difference equation (2) are imposed by fixing the values

$$
y_{0}, \ldots, y_{k_{1}-1}, y_{N}, \ldots, y_{N+k_{2}-1} \text {, }
$$

where $k_{1}$ and $k_{2}$ are two integers, $k_{1}+k_{2}=k$. In this way, one fixes the first $k_{1}$ and the final $k_{2}$ values of the discrete solution. This means that the continuous IVP is now approximated by means of a discrete BVP. The methods obtained in this way have been called boundary value methods (BVMs). If the values (3) are fixed, we say that scheme (2) is used with $\left(k_{1}, k_{2}\right)$ boundary conditions $[9,10]$. As before, only the value $y_{0}$ is provided by the continuous problem, while the remaining values must be obtained in some way.

The definition of 0 -stability and absolute stability for IVMs are now generalized to BVMs by introducing the following two kinds of polynomials.

Definition 1. A polynomial $p(z)$ of degree $k=k_{1}+k_{2}$ is said to be an $S_{k_{1} k_{2}}$-polynomial if its roots are such that

$$
\left|z_{1}\right| \leqslant\left|z_{2}\right| \leqslant \cdots \leqslant\left|z_{k_{1}}\right|<1<\left|z_{k_{1}+1}\right| \leqslant \cdots \leqslant\left|z_{k}\right|
$$

while it is called an $N_{k_{1} k_{2}}$-polynomial if

$$
\left|z_{1}\right| \leqslant\left|z_{2}\right| \leqslant \cdots \leqslant\left|z_{k_{1}}\right| \leqslant 1<\left|z_{k_{1}+1}\right| \leqslant \cdots \leqslant\left|z_{k}\right| \text {, }
$$

where the roots of unit modulus are assumed to be simple. 
We observe that for $k_{1}=k$ and $k_{2}=0, N_{k_{1} k_{2}}$-polynomials reduce to Von Neumann polynomials while $S_{k_{1} k_{2}}$-polynomials reduce to Schur polynomials. Now we can give the following definitions for BVMs $[9,10]$.

Definition 2. BVM (2) used with $\left(k_{1}, k_{2}\right)$-boundary conditions is said to be $0_{k_{1} k_{2}}$-stable, if the polynomial

$$
\rho(z)=\sum_{i=0}^{k} \alpha_{i} z^{i}
$$

is an $N_{k_{1} k_{2}}$-polynomial. It is said to be $\left(k_{1}, k_{2}\right)$-absolutely stable for a given $q \in \mathbb{C}$ if the polynomial

$$
\pi(z, q)=\sum_{i=0}^{k}\left(\alpha_{i}-q \beta_{i}\right) z^{i}
$$

is an $S_{k_{1} k_{2}}$-polynomial. The region

$$
D_{k_{1} k_{2}}=\left\{q \in \mathbb{C}: \pi(z, q) \text { is an } S_{k_{1} k_{2}} \text {-polynomial }\right\}
$$

is called the region of $\left(k_{1}, k_{2}\right)$-absolute stability of the method. Finally, the method is said to be $A_{k_{1} k_{2}}$ stable if $\mathbb{C}^{-} \subseteq D_{k_{1} k_{2}}$, where $\mathbb{C}^{-}$is the left half complex plane.

The given definitions reduce to the well-known ones for IVMs when $k_{1}=k$ and $k_{2}=0$. This means that the class of the BVMs contains the IVMs as a subclass.

For the moment we shall neglect the problem of finding the unknown values in (3). This problem will be considered in Section 4.

The advantage of the BVMs over the IVMs is that now there are no more barriers concerning the order of $0_{k_{1} k_{2}}$-stable and $A_{k_{1} k_{2}}$-stable methods. In fact there are $0_{k_{1} k_{2}}$-stable and $\mathrm{A}_{k_{1} k_{2}}$-stable methods of order up to $2 k[1,10]$ for every odd value of $k$.

\section{Use of the BVMs for approximating continuous BVPs}

Since the BVMs consists in approximating a continuous initial value problem by means of a discrete boundary value one, they are natural candidates to approximate continuous boundary value problems, with some slight modification. For simplicity, we shall consider only two-point BVPs, but the generalization to multipoint BVPs is straightforward.

Then, let us consider the scalar problem,

$$
y^{\prime}=\lambda y, \quad b_{1} y(0)+b_{2} y(T)=\eta,
$$

whose solution is given by:

$$
y(t)=\mathrm{e}^{\lambda t} \frac{\eta}{b_{1}+b_{2} \mathrm{e}^{\lambda T}} .
$$


Unlike the case of IVPs, where one usually assumes $\mathfrak{R}(\lambda)<0$, here $\mathfrak{R}(\lambda)$ may be either positive or negative. This because in a general well-conditioned BVP both increasing and decreasing modes must be present (dichotomy) [6,12].

Now, let us consider the approximation provided by a $k$-step LMM:

$$
\sum_{i=0}^{k}\left(\alpha_{i}-q \beta_{i}\right) y_{n+i}=0, \quad n=0, \ldots, N-k_{1}-1,
$$

where, as usual, $q=h \lambda$, and the stepsize is $h=T /\left(N+k_{2}-1\right), k_{1}+k_{2}=k$. The $k$ conditions needed by the discrete scheme are now given by fixing the following $k-1$ values of the discrete solution

$$
y_{1}, \ldots, y_{k_{1}-1}, y_{N}, \ldots, y_{N+k_{2}-1},
$$

while the remaining condition is obtained by the continuous problem:

$$
b_{1} y_{0}+b_{2} y_{N+k_{2}-1}=\eta \text {. }
$$

In analogy with the case of the methods for IVPs, we shall say that scheme (7) is used with $\left(k_{1}, k_{2}\right)$-boundary conditions. We shall study the solution of the discrete problem (7)-(9) under some assumptions on the roots of the characteristic polynomial associated with (7).

Theorem 3. Let $z_{1}, \ldots, z_{k}$ be the roots of the stability polynomial (4) associated with the difference equation (7), which we shall assume ordered by increasing moduli:

$$
\left|z_{1}\right| \leqslant \cdots \leqslant\left|z_{k}\right| \text {. }
$$

Moreover, assume that

(1) it holds that

$$
\left|z_{k_{1}-1}\right|<\left|z_{k_{1}}\right|<\left|z_{k_{1}+1}\right|, \quad\left|z_{k_{1}-1}\right|<1<\left|z_{k_{1}+1}\right| \text {; }
$$

(2) $z_{k_{1}}$ is the principal root of the method, whose order is $p \geqslant 1$;

(3) the additional values in (8) are $\mathrm{O}\left(h^{p}\right)$ approximations of the corresponding values $y\left(t_{1}\right), \ldots, y\left(t_{k_{1}-1}\right), y\left(t_{N}\right), \ldots, y\left(t_{N+k_{2}-1}\right)$ of the continuous solution.

It follows that, for $n=k_{1}, \ldots, N-1$, one has $y_{n}=y\left(t_{n}\right)+\mathrm{O}\left(h^{p}\right)$.

Proof. Let us suppose the roots $\left\{z_{i}\right\}$ to be distinct, for simplicity. Then the discrete solution of (7) is given by

$$
y_{n}=c_{k_{1}} z_{k_{1}}^{n}+\boldsymbol{e}_{\mathrm{i}}^{\mathrm{T}} D_{\mathrm{i}}^{n} c_{\mathrm{i}}+\boldsymbol{e}_{\mathrm{f}}^{\mathrm{T}} D_{\mathrm{f}}^{n} c_{\mathrm{f}},
$$

where

$$
\begin{array}{ll}
D_{\mathrm{i}}=\left(\begin{array}{ccc}
z_{1} & & \\
& \ddots & \\
& & z_{k_{1}-1}
\end{array}\right), & D_{\mathrm{f}}=\left(\begin{array}{lll}
z_{k_{1}+1} & & \\
& \ddots & \\
& & z_{k}
\end{array}\right), \\
\boldsymbol{e}_{\mathrm{i}}=(1, \ldots, 1)_{k_{1}-1}^{\mathrm{T}}, & \boldsymbol{e}_{\mathrm{f}}=(1, \ldots, 1)_{k_{2}}^{\mathrm{T}}, \\
\boldsymbol{c}_{\mathrm{i}}=\left(c_{1}, \ldots, c_{k_{1}-1}\right)^{\mathrm{T}}, & \boldsymbol{c}_{\mathrm{f}}=\left(c_{k_{1}+1}, \ldots, c_{k}\right)^{\mathrm{T}} .
\end{array}
$$


The constants $c_{k_{1}}, c_{\mathrm{i}}$ and $c_{\mathrm{f}}$ are determined by imposing the conditions (8) and (9):

$$
\hat{G}\left(\begin{array}{c}
c_{k_{1}} \\
c_{\mathrm{i}} \\
c_{\mathrm{f}}
\end{array}\right)=\left(\begin{array}{c}
\eta \rho \\
y_{\mathrm{i}} \\
y_{\mathrm{f}}
\end{array}\right)
$$

where $\rho=\left(b_{1}+b_{2} z_{k 1}^{N+k_{2}-1}\right)^{-1}$,

$$
\begin{aligned}
& \hat{G}=\left(\begin{array}{ccc}
1 & s_{\mathrm{i}}^{\mathrm{T}} & \boldsymbol{s}_{\mathrm{f}}^{\mathrm{T}} \\
w_{k_{1}-1} z_{k_{1}}, & U_{k_{1}-1} D_{\mathrm{i}} & V_{k_{1}-1} D_{\mathrm{f}} \\
w_{k_{2}} z_{k_{1}}^{N} & U_{k_{2}} D_{\mathrm{i}}^{N} & V_{k_{2}} D_{\mathrm{f}}^{N}
\end{array}\right), \\
& s_{\mathrm{i}}=\rho\left(b_{1} I_{k_{1}-1}+b_{2} D_{\mathrm{i}}^{N+k_{2}-1}\right) e_{\mathrm{i}}, \quad s_{\mathrm{f}}=\rho\left(b_{1} I_{k_{2}}+b_{2} D_{\mathrm{f}}^{N+k_{2}-1}\right) \boldsymbol{e}_{\mathrm{f}} \text {, } \\
& U_{j}=\left(\begin{array}{ccc}
1 & \ldots & 1 \\
z_{1} & \ldots & z_{k_{1}-1} \\
\vdots & & \vdots \\
z_{1}^{j-1} & \ldots & z_{k_{1}-1}^{j-1}
\end{array}\right), \quad V_{j}=\left(\begin{array}{ccc}
1 & \ldots & 1 \\
z_{k_{1}+1} & \ldots & z_{k} \\
\vdots & & \vdots \\
z_{k_{1}+1}^{j-1} & \ldots & z_{k}^{j-1}
\end{array}\right) \text {, } \\
& \boldsymbol{w}_{j}=\left(1, z_{k_{1}}, \ldots, z_{k_{1}}^{j-1}\right)^{\mathrm{T}} \\
& \boldsymbol{y}_{\mathrm{i}}=\left(y_{1}, \ldots, y_{k_{1}-1}\right)^{\mathrm{T}}, \quad \boldsymbol{y}_{\mathrm{f}}=\left(y_{N}, \ldots, y_{N+k_{2}-1}\right)^{\mathrm{T}} .
\end{aligned}
$$

We observe that all the leading principal submatrices of the matrix $\hat{G}$ can be proved to be nonsingular, under the assumptions (11) (the long proof of this result can be found in [10]). Therefore, we can consider the following block LU factorization of $\hat{G}$,

$$
\hat{G}=\left(\begin{array}{ccc}
1 & & \\
w_{k_{1}-1} z_{k_{1}} & I_{k_{1}-1} & \\
\boldsymbol{w}_{k_{2}} z_{k_{1}}^{N} & H & I_{k_{2}}
\end{array}\right)\left(\begin{array}{ccc}
1 & s_{\mathrm{i}}^{\mathrm{T}} & \boldsymbol{s}_{\mathrm{f}}^{\mathrm{T}} \\
& B_{1} & B_{2} \\
& & C
\end{array}\right)
$$

where $I_{k_{1}-1}$ and $I_{k_{2}}$ are the identity matrices of size $k_{1}-1$ and $k_{2}$, respectively,

$$
\begin{aligned}
& B_{1}=U_{k_{1}-1} D_{\mathrm{i}}-z_{k_{1}} \boldsymbol{w}_{k_{1}-1} \boldsymbol{s}_{\mathrm{i}}^{\mathrm{T}}, \quad B_{2}=V_{k_{1}-1} D_{\mathrm{f}}-z_{k_{1}} \boldsymbol{w}_{k_{1}-1} \boldsymbol{s}_{\mathrm{f}}^{\mathrm{T}}, \\
& H=\left(U_{k_{2}} D_{\mathrm{i}}^{N}-z_{k_{1}}^{N} \boldsymbol{w}_{k_{2}} \boldsymbol{s}_{\mathrm{i}}^{\mathrm{T}}\right) B_{1}^{-1}=\mathrm{O}\left(\left|z_{k_{1}}\right|^{N}\right), \\
& C=V_{k_{2}} D_{\mathrm{f}}^{N}-z_{k_{1}}^{N} \boldsymbol{w}_{k_{2}} \boldsymbol{s}_{\mathrm{f}}^{\mathrm{T}}-H B_{2}=\left(V_{k_{2}}-z_{k_{1}}^{N} \boldsymbol{w}_{k_{2}} \boldsymbol{s}_{\mathrm{f}}^{\mathrm{T}}+\mathrm{O}\left(\left|z_{k_{1}} / z_{k_{1}+1}\right|^{N}\right)\right) D_{\mathrm{f}}^{N},
\end{aligned}
$$

then one obtains:

$$
\hat{G}^{-1}=\left(\begin{array}{ccc}
1+z_{k_{1}} \boldsymbol{s}_{\mathrm{i}}^{\mathrm{T}} B_{1}^{-1} \boldsymbol{w}_{k_{1}-1}+\boldsymbol{u}^{\mathrm{T}} C^{-1} \boldsymbol{v} & \boldsymbol{u}^{\mathrm{T}} C^{-1} H-s_{\mathrm{i}}^{\mathrm{T}} B_{1}^{-1} & -\boldsymbol{u}^{\mathrm{T}} C^{-1} \\
B_{1}^{-1}\left(B_{2} C^{-1} v-z_{k_{1}} \boldsymbol{w}_{k_{1}-1}\right) & B_{1}^{-1}\left(I_{k_{1}-1}+B_{2} C^{-1} H\right) & -B_{1}^{-1} B_{2} C^{-1} \\
-C^{-1} v & -C^{-1} H & C^{-1}
\end{array}\right),
$$


where

$$
u^{\mathrm{T}}=\boldsymbol{s}_{\mathrm{f}}^{\mathrm{T}}-s_{\mathrm{i}}^{\mathrm{T}} B_{1}^{-1} B_{2}, \quad v=z_{k_{1}}^{N} \boldsymbol{w}_{k_{2}}-z_{k_{1}} H \boldsymbol{w}_{k_{1}-1} .
$$

It follows that

$$
\begin{aligned}
& c_{k_{1}}=\left(1+z_{k_{1}} s_{\mathrm{i}}^{\mathrm{T}} B_{1}^{-1} \boldsymbol{w}_{k_{1}-1}+u^{\mathrm{T}} C^{-1} v\right) \eta \rho+\left(u^{\mathrm{T}} C^{-1} H-s_{\mathrm{i}}^{\mathrm{T}} B_{1}^{-1}\right) y_{\mathrm{i}}-\left(u^{\mathrm{T}} C^{-1}\right) y_{\mathrm{f}} \\
& =\eta \rho+\left(u^{\mathrm{T}} C^{-1} H-s_{\mathrm{i}}^{\mathrm{T}} B_{1}^{-1}\right)\left(y_{\mathrm{i}}-\eta \rho z_{k_{1}} \boldsymbol{w}_{k_{1}-1}\right)-\boldsymbol{u}^{\mathrm{T}} C^{-1}\left(y_{\mathrm{f}}-\eta \rho z_{k_{1}}^{N_{1}} \boldsymbol{w}_{k_{2}}\right) \text {, } \\
& c_{\mathrm{i}}=B_{1}^{-1}\left(\left(B_{2} C^{-1} v-z_{k_{1}} \boldsymbol{w}_{k_{1}-1}\right) \eta \rho+\left(I_{k_{1}-1}+B_{2} C^{-1} H\right) \boldsymbol{y}_{\mathrm{i}}-B_{2} C^{-1} \boldsymbol{y}_{\mathrm{f}}\right) \\
& =B_{1}^{-1}\left(\left(I_{k_{1}-1}+B_{2} C^{-1} H\right)\left(y_{\mathrm{i}}-\eta \rho z_{k_{1}} \boldsymbol{w}_{k_{1}-1}\right)-B_{2} C^{-1}\left(\boldsymbol{y}_{\mathrm{f}}-\eta \rho z_{k_{1}}^{N_{k_{2}}}\right)\right), \\
& c_{\mathrm{f}}=C^{-1}\left(y_{\mathrm{f}}-v \eta \rho-H y_{\mathrm{i}}\right) \\
& =C^{-1}\left(\left(y_{\mathrm{f}}-\eta \rho z_{k_{1}}^{N_{k_{2}}}\right)-H\left(y_{\mathrm{i}}-\eta \rho z_{k_{1}} \boldsymbol{w}_{k_{1}-1}\right)\right) \text {. }
\end{aligned}
$$

Now, if $z_{k_{1}}$ is the root which approximates the exponential, and the discrete scheme has order $p \geqslant 1$, then it follows that $z_{k_{1}}=\mathrm{e}^{q}+\mathrm{O}\left(h^{p+1}\right)$ and, therefore, one obtains

$$
\left(y_{\mathrm{i}}-\eta \rho z_{k_{1}} \boldsymbol{w}_{k_{1}-1}\right)=\mathrm{O}\left(h^{p}\right), \quad\left(\boldsymbol{y}_{\mathrm{f}}-\eta \rho z_{k_{1}}^{N} \boldsymbol{w}_{k_{2}}\right)=\mathrm{O}\left(h^{p}\right)
$$

provided that $y_{\mathrm{i}}$ and $\boldsymbol{y}_{\mathrm{f}}$ are at least $\mathrm{O}\left(h^{p}\right)$ accurate. From these relations and the relations (6) and (12) finally it follows that

$$
y_{n}=\eta \rho z_{k_{1}}^{n}+\mathrm{O}\left(h^{p}\right)=y\left(t_{n}\right)+\mathrm{O}\left(h^{p}\right),
$$

that is, the discrete solution approximates the continuous solution with a global error $\mathrm{O}\left(h^{p}\right)$.

We can conclude that a BVM with $\left(k_{1}, k_{2}\right)$-boundary conditions can be used to approximate the solution of the BVP (5), provided that the root $z_{k_{1}}$ of the characteristic polynomial associated with the difference equation is the one which approximates the exponential. For this reason it is obvious that the natural candidates to approximate continuous BVPs are the BVMs having $D_{k_{1} k_{2}} \equiv \mathbb{C}^{-}$. There are many such methods; in particular, we consider the class of the extended trapezoidal rules (ETRs) [3,10] and the top order methods (TOMs) [1,10]. Let us briefly recall these methods.

ETRs are methods with $k=2 \nu-1$ steps having the form:

$$
y_{n}-y_{n-1}=h \sum_{i=-\nu}^{\nu-1} \beta_{i+\nu} f_{n+i}, \quad n=\nu, \ldots, N-1 .
$$

The coefficients are determined so that the scheme has order $k+1$. Its use is with $(\nu, \nu-1)$ boundary conditions. The TOM with the same number of steps,

$$
\sum_{i=-\nu}^{\nu-1} \alpha_{i+\nu} y_{n+i}=h \sum_{i=-\nu}^{\nu-1} \beta_{i+\nu} f_{n+i}, \quad n=\nu, \ldots, N-1,
$$

has order $2 k$. This method must also be used with $(\nu, \nu-1)$-boundary conditions. 


\section{Implementation of the methods and numerical examples}

In this section we shall study the implementation of the above-mentioned BVMs. In fact, if the formula (13) or (14) is used with $(\nu, \nu-1)$-boundary conditions and a stepsize $h=(b-a)$ / $(N+\nu-2)$ to approximate the BVP

$$
y^{\prime}=f(t, y), \quad t \in[a, b], \quad g(y(a), y(b))=\eta,
$$

then the following values of the discrete solution,

$$
y_{1}, \ldots, y_{\nu-1}, y_{N}, \ldots, y_{N+\nu-2},
$$

must be imposed along with the boundary condition

$$
g\left(y_{0}, y_{N+\nu-2}\right)=\eta \text {. }
$$

Moreover, in order to have a solution with accuracy $\mathrm{O}\left(h^{p}\right)$, if $p$ is the order of the used method (main method), the approximations (16) need to be at least $\mathrm{O}\left(h^{p}\right)$ accurate. If these values are known, then scheme (13) or (14), used for $n=\nu, \ldots, N-1$, along with the boundary condition (17), provides a set of $N-\nu+1$ equations in the $N-\nu+1$ unknowns

$$
y_{0}, y_{\nu}, \ldots, y_{N-1} \text {, }
$$

so that the problem can be solved. However, since the $2 \nu-2$ values (16) are usually not available, we must treat them as unknowns, by adding a set of $2 \nu-2$ equations independent of the previously considered ones. This is done by considering a set of additional methods, whose order must be at least $p-1$, that is with an $\mathrm{O}\left(h^{p}\right)$ truncation error, if $p$ is the order of the chosen main method.

\subsection{Use of the ETRs}

If one use scheme (13) for $n=\nu, \ldots, N-1$, the following $\nu-1$ equations:

$$
y_{r}-y_{r-1}=h \sum_{i=0}^{2 \nu-2} \beta_{i, r} f_{i}, \quad r=1, \ldots, \nu-1,
$$

are used to implicitly impose the first $\nu-1$ values in (16). In order to preserve the global order $k+1$ of the main method, the coefficients $\left\{\beta_{i, r}\right\}$ are chosen by requiring those equations to have a truncation error $\mathrm{O}\left(h^{k+1}\right)$. The final $\nu-1$ values are implicitly imposed by using the following additional equations:

$$
y_{r}-y_{r-1}=h \sum_{i=N-\nu+1}^{N+\nu-2} \beta_{i-N+\nu-1, r} f_{i}, \quad r=N, \ldots, N+\nu-2,
$$

where the coefficients $\left\{\beta_{i, r}\right\}$ are chosen again by imposing the truncation error of each equation to be $\mathrm{O}\left(h^{k+1}\right)$.

Example 1. The trapezoidal rule, which is the simplest ETR, does not need additional equations, since it requires only the boundary condition. 
The ETR of order 4,

$$
y_{n}-y_{n-1}=\frac{1}{24} h\left(-f_{n-2}+13 f_{n-1}+13 f_{n}-f_{n+1}\right), \quad n=2, \ldots, N-1,
$$

requires the two additional equations:

$$
\begin{aligned}
& y_{1}-y_{0}=\frac{1}{12} h\left(5 f_{0}+8 f_{1}-f_{2}\right), \\
& y_{N}-y_{N-1}=\frac{1}{12} h\left(-f_{N-2}+8 f_{N-1}+5 f_{N}\right) .
\end{aligned}
$$

They are obtained from the reverse Adams formula [2,10] and the Adams-Moulton formula of order 3 , respectively.

The ETR of order 6 ,

$$
\begin{aligned}
& y_{n}-y_{n-1}=\frac{1}{1440} h\left(11 f_{n-3}-93 f_{n-2}+802 f_{n-1}+802 f_{n}-93 f_{n+1}+11 f_{n+2}\right), \\
& n=3, \ldots, N-1,
\end{aligned}
$$

requires the four additional equations:

$$
\begin{aligned}
& y_{1}-y_{0}=\frac{1}{720} h\left(251 f_{0}+646 f_{1}-264 f_{2}+106 f_{3}-19 f_{4}\right), \\
& y_{2}-y_{1}=\frac{1}{720} h\left(-19 f_{0}+346 f_{1}+456 f_{2}-74 f_{3}+11 f_{4}\right), \\
& y_{N}-y_{N-1}=\frac{1}{720} h\left(11 f_{N-3}-74 f_{N-2}+456 f_{N-1}+346 f_{N}-19 f_{N+1}\right), \\
& y_{N+1}-y_{N}=\frac{1}{720} h\left(-19 f_{N-3}+106 f_{N-2}-264 f_{N-1}+646 f_{N}+251 f_{N+1}\right) .
\end{aligned}
$$

\subsection{Use of the TOMs}

If one uses scheme (14) for $n+\nu, \ldots, N-1$, then there are essentially two ways of choosing the additional methods to impose the additional $k-1=2 \nu-2$ conditions needed by the discrete problem. The first way is the use of different methods of order at least $2 k-1$ (to preserve the order $2 k$ of the main method) to derive each equation, as it has been done for the ETRs. Alternatively one may consider a fixed method of order $2 k-1$ used on different grid points. Each way has it own advantages and drawbacks. Let us examine the two cases.

In the first case, one may use the following Adams-type methods

$$
y_{r}-y_{r-1}=h \sum_{i=0}^{2 k-2} \beta_{i, r} f_{i}, \quad r=1, \ldots, \nu-1,
$$

to obtain the initial additional equations and

$$
y_{r}-y_{r-1}=h \sum_{i=0}^{2 k-2} \beta_{i, r} f_{N+\nu-2-i}, \quad r=N, \ldots, N+\nu-2,
$$


to obtain the final additional ones. For every value of $r$, the coefficients $\left\{\beta_{i, r}\right\}$ are chosen in order for the formula to have order $2 k-1$.

In the second case, one fixes two methods of order $2 k-1$ which are used on different grid points, one to obtain the initial additional equations, the other for the final ones. A good candidate is obtained by deriving a method of order $2 k-1$ having the same number $k$ of steps of the used TOM.

An advantage of the latter approach over the former one is its compactness, due to the fact that the used additional method has the smallest number of steps. On the other hand, in general the last approach produces much more ill-conditioned linear systems, when solving the complete set of equations, with respect to those produced by using the first approach. This last feature is more evident for $\nu>2$.

Example 2. The TOM of order 2, that is the trapezoidal rule, does not require additional equations, as we have already seen in the previous example.

The TOM of order 6 ,

$$
\frac{11}{27} y_{n+1}+y_{n}-y_{n-1}-\frac{11}{27} y_{n-2}=h\left(\frac{1}{9} f_{n+1}+f_{n}+f_{n-1}+\frac{1}{9} f_{n-2}\right), \quad n=2, \ldots, N-1,
$$

requires two addition equations. They are conveniently obtained by using the second of the previously mentioned possibilities:

$$
\begin{aligned}
& \frac{25}{108} y_{3}+y_{2}-\frac{3}{4} y_{1}-\frac{13}{27} y_{0}=h\left(\frac{1}{18} f_{3}+\frac{3}{4} f_{2}+f_{1}+\frac{5}{36} f_{0}\right), \\
& \frac{13}{27} y_{N}+\frac{3}{4} y_{N-1}-y_{N-2}-\frac{25}{108} y_{N-3}=h\left(\frac{5}{36} f_{N}+f_{N-1}+\frac{3}{4} f_{N-2}+\frac{1}{18} f_{N-3}\right),
\end{aligned}
$$

obtained by two methods of order 5 .

The TOM of order 10

$$
\begin{aligned}
& \frac{137}{3000} y_{n+2}+\frac{13}{24} y_{n+1}+\frac{2}{3} y_{n}-\frac{2}{3} y_{n-1}-\frac{13}{24} y_{n-2}-\frac{137}{3000} y_{n-3} \\
& =h\left(\frac{1}{100} f_{n+2}+\frac{1}{4} f_{n+1}+f_{n}+f_{n-1}+\frac{1}{4} f_{n-2}+\frac{1}{100} f_{n-3}\right), \quad N=3, \ldots, N-1,
\end{aligned}
$$

needs four additional equations that can be obtained by using four additional Adams-type methods of order 9 like (20) and (21):

$$
\begin{aligned}
y_{1}-y_{0}=h( & -\frac{33953}{3628800} f_{8}+\frac{156437}{1814400} f_{7}-\frac{645607}{1814400} f_{6}+\frac{1573169}{1814400} f_{5} \\
& \left.-\frac{31457}{22680} f_{4}+\frac{2797679}{1814400} f_{3}-\frac{2302297}{1814400} f_{2}+\frac{2233547}{1814400} f_{1}+\frac{1070017}{3628800} f_{0}\right), \\
y_{2}-y_{1}= & h\left(\frac{7297}{3628800} f_{8}-\frac{34453}{1814400} f_{7}+\frac{147143}{1814400} f_{6}-\frac{377521}{1814400} f_{5}\right. \\
& \left.+\frac{8233}{22680} f_{4}-\frac{876271}{1814400} f_{3}+\frac{1622393}{1814400} f_{2}+\frac{687797}{1814400} f_{1}-\frac{33953}{3628800} f_{0}\right),
\end{aligned}
$$


and

$$
\begin{aligned}
y_{N}-y_{N-1}=h( & \frac{7297}{3628800} f_{N-7}-\frac{34453}{1814400} f_{N-6}+\frac{147143}{1814400} f_{N-5} \\
& -\frac{377521}{1814400} f_{N-4}+\frac{8233}{22680} f_{N-3}-\frac{876271}{1814400} f_{N-2} \\
& \left.+\frac{1622393}{1814400} f_{N-1}+\frac{687797}{1814400} f_{N}-\frac{33953}{3628800} f_{N+1}\right), \\
y_{N+1}-y_{N}=h(- & \frac{33953}{3628800} f_{N-7}+\frac{156437}{1814400} f_{N-6}-\frac{645607}{1814400} f_{N-5} \\
& +\frac{1573169}{1814400} f_{N-4}-\frac{31457}{22680} f_{N-3}+\frac{2797679}{1814400} f_{N-2}-\frac{2302297}{1814400} f_{N-1} \\
& \left.+\frac{2233547}{1814400} f_{N}+\frac{1070017}{3628800} f_{N+1}\right) .
\end{aligned}
$$

Alternatively, one can use only two methods of order 9, but on different grid points:

$$
\begin{aligned}
& \frac{49}{48000} y_{r+5}+\frac{29}{1728} y_{r+4}+\frac{13}{432} y_{r+3}-\frac{1}{48} y_{r+2}-\frac{85}{3456} y_{r+1}-\frac{533}{216000} y_{r} \\
& =h\left(\frac{1}{4800} f_{r+5}+\frac{1}{144} f_{r+4}+\frac{5}{144} f_{r+3}+\frac{1}{24} f_{r+2}+\frac{7}{576} f_{r+1}+\frac{1}{1800} f_{r}\right), \quad r=0,1,
\end{aligned}
$$

and

$$
\begin{aligned}
& \frac{533}{216000} y_{r}+\frac{85}{3456} y_{r-1}+\frac{1}{48} y_{r-2}-\frac{13}{432} y_{r-3}-\frac{29}{1728} y_{r-4}-\frac{49}{48000} y_{r-5} \\
& \quad=h\left(\frac{1}{1800} f_{r}+\frac{7}{576} f_{r-1}+\frac{1}{24} f_{r-2}+\frac{5}{144} f_{r-3}+\frac{1}{144} f_{r-4}+\frac{1}{4800} f_{r-5}\right), \\
& r=N, N+1 .
\end{aligned}
$$

It is evident that the second way is more compact than the first one. However, as said before, the linear systems obtained when solving Eqs. (22), (17), (23)-(26) are generally much more better conditioned than those resulting from the solution of Eqs. (22), (17), (27)-(28).

\subsection{Numerical examples}

In the following some numerical examples are provided. They are obtained by approximating continuous BVPs with the ETR of order 4 and the TOM of order 6 examined in Examples 1 and 2.

Example 3. Consider the problem [6]

$$
\begin{aligned}
& u^{\prime \prime}-4 u=16 t+12 t^{2}-4 t^{4}, \quad 0<t<1, \\
& u(0)=u^{\prime}(1)=0
\end{aligned}
$$




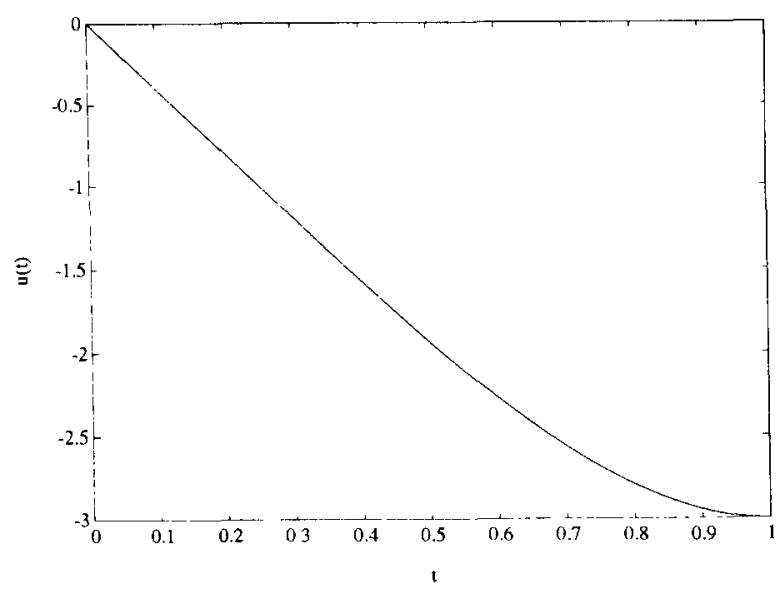

Fig. 1. Solution of problem (29).

Table 1

Maximum errors for various stepsizes (problem (29))

\begin{tabular}{llll}
\hline$h$ & \multicolumn{2}{l}{ ETR of order 4} & TOM of order 6 \\
\cline { 2 - 3 } & $\|e\|_{\infty}$ & rate & $\|e\|_{\infty}$ \\
\hline 0.25 & $2.628 \mathrm{e}-3$ & - & $1.776 \mathrm{e}-15$ \\
0.125 & $1.955 \mathrm{e}-4$ & 3.75 & $1.776 \mathrm{e}-15$ \\
0.0625 & $1.359 \mathrm{e}-5$ & 3.85 & $1.332 \mathrm{e}-15$ \\
0.03125 & $8.989 \mathrm{e}-7$ & 3.92 & $2.664 \mathrm{e}-15$ \\
0.015625 & $5.785 \mathrm{e}-8$ & 3.96 & $6.661 \mathrm{e}-15$ \\
\hline
\end{tabular}

whose solution $u(t)=t^{4}-4 t$ is plotted in Fig. 1. In Table 1 the maximum errors are reported for various stepsizes, by using the above mentioned methods.

In the case of the TOM of order 6 , the errors are of the order of the machine precision and, therefore, they are essentially due to round-off errors more than to the truncation errors. For this reason, in this case it is not possible to compute the rate of convergence.

Example 4. Consider the problem [6]

$$
\begin{aligned}
& \left(t^{3} u^{\prime \prime}\right)^{\prime \prime}=1, \quad 1<t<2, \\
& u(1)=u^{\prime \prime}(1)=u(2)=u^{\prime \prime}(2)=0,
\end{aligned}
$$

whose solution $u(t)=\frac{1}{4}(10 \log (2)-3)(1-t)+\frac{1}{2}\left(t^{-1}+(3+t) \log (t)-t\right)$ is plotted in Fig. 2. In Table 2 the maximum errors are reported for various stepsizes.

Example 5. Consider the singular perturbation problem [4]

$$
\begin{aligned}
& \varepsilon u^{\prime \prime}+t u^{\prime}=-\varepsilon \pi^{2} \cos (\pi t)-\pi t \sin (\pi t), \quad-1<t<1, \\
& u(-1)=-2, \quad u(1)=0,
\end{aligned}
$$




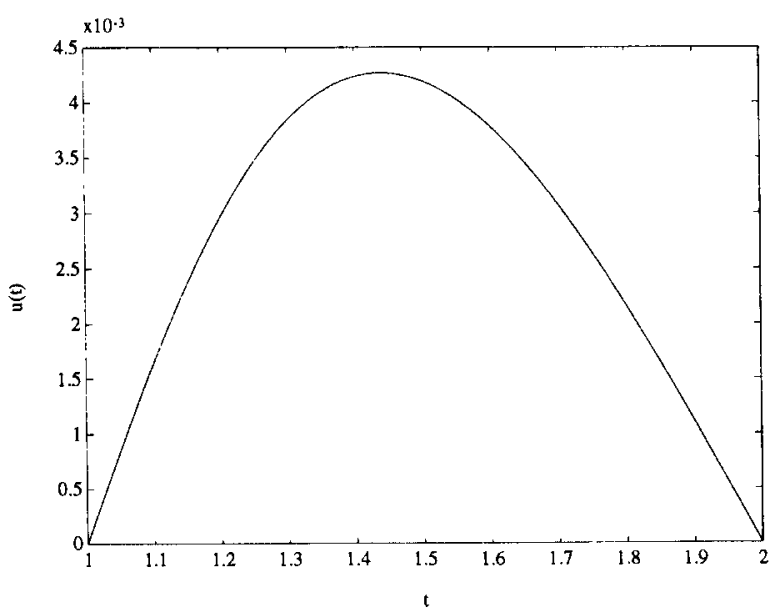

Fig. 2. Solution of problem (30).

Table 2

Maximum errors for various stepsizes (problem (30))

\begin{tabular}{llllll}
\hline$h$ & \multicolumn{2}{l}{ ETR of order 4 } & & \multicolumn{2}{l}{ TOM of order 6 } \\
\cline { 2 - 3 } & $\|e\|_{\infty}$ & rate & & $\|e\|_{\infty}$ & rate \\
\hline 0.25 & $1.092 \mathrm{e}-04$ & - & $2.014 \mathrm{e}-04$ & - \\
0.125 & $1.004 \mathrm{e}-05$ & 3.44 & $2.014 \mathrm{e}-06$ & 6.64 \\
0.0625 & $1.290 \mathrm{e}-06$ & 2.96 & $5.917 \mathrm{e}-08$ & 5.09 \\
0.03125 & $1.133 \mathrm{e}-07$ & 3.51 & $1.392 \mathrm{e}-09$ & 5.41 \\
0.015625 & $8.387 \mathrm{e}-09$ & 3.76 & $2.710 \mathrm{e}-11$ & 5.68 \\
0.0078125 & $5.705 \mathrm{e}-10$ & 3.88 & $4.740 \mathrm{e}-13$ & 5.84 \\
0.00390625 & $3.720 \mathrm{e}-11$ & 3.94 & $7.957 \mathrm{e}-15$ & 5.90 \\
\hline
\end{tabular}

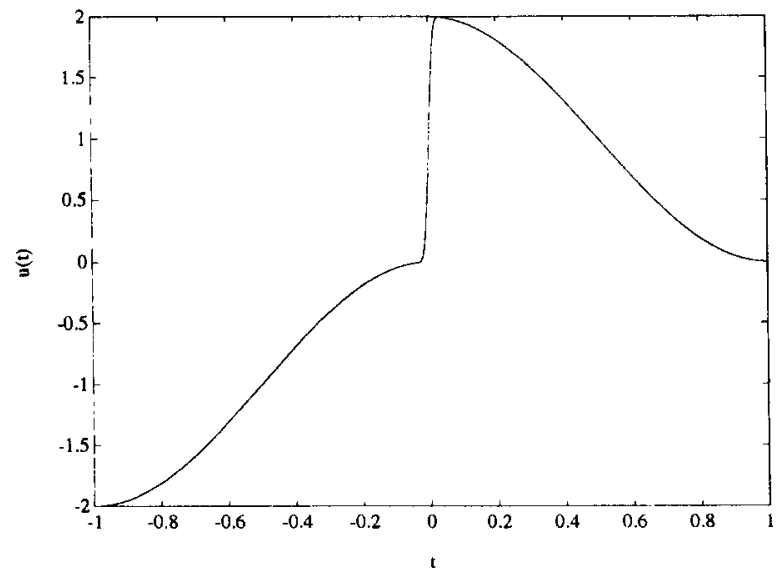

Fig. 3. Solution of problem (31). 
Table 3

Maximum errors for various step sizes (problem (31))

\begin{tabular}{|c|c|c|c|c|}
\hline \multirow[t]{2}{*}{$h$} & \multicolumn{2}{|c|}{ ETR of order 4} & \multicolumn{2}{|c|}{ TOM of order 6} \\
\hline & $\|\boldsymbol{\| e}\|_{\infty}$ & $\overline{\text { rate }}$ & $\overline{\|e\|_{x}}$ & \\
\hline$\overline{0.01}$ & $1.860 \mathrm{e}-2$ & - & $2.980 \mathrm{e}-3$ & - \\
\hline 0.05 & $1.515 \mathrm{e}-3$ & 3.62 & $5.729 \mathrm{e}-5$ & 5.70 \\
\hline 0.00250 & $1.080 \mathrm{e}-4$ & 3.81 & $7.382 \mathrm{e}-7$ & 6.28 \\
\hline 0.00125 & $6.839 \mathrm{e}-6$ & 3.98 & $1.114 \mathrm{e}-8$ & 6.05 \\
\hline
\end{tabular}

where $\varepsilon=10^{-4}$, whose solution $u(t)=\cos (\pi t)+\operatorname{erf}(t / \sqrt{2 \varepsilon}) / \operatorname{erf}(1 / \sqrt{2 \varepsilon})$ is plotted in Fig. 3 . In Table 3 the maximum errors are reported for various stepsizes.

Example 6. Consider the nonlinear problem due to Troesch [14]

$$
\begin{array}{ll}
u^{\prime \prime}=\lambda \sinh (\lambda u), & 0<t<1, \\
u(0)=0, & u(1)=1 .
\end{array}
$$

The solution is plotted in Fig. 4, for $\lambda=5$. A straight implementation of the Newton method, starting from a zero initial approximation, has been used for both the two methods. In Table 4 the maximum errors are reported for various stepsizes.

Problem (31) is significant, since (see Fig. 3) the solution has a layer at $t=0$. In this case, one cannot expect a uniform mesh to be the best choice. In fact, in Fig. 5 it is plotted the absolute error for the ETR of order 4 (but similar considerations hold true for the TOM of order 6). As one may expect, the larger errors are near the layer, where a smaller stepsize should be used. However, if one uses the error equidistribution technique described in [6] the original mesh is sensibly improved (see also [10]). In fact, after one equidistribution over the original uniform mesh with $h=0.01$, one obtains the mesh shown in Fig. 6. In the figure it is

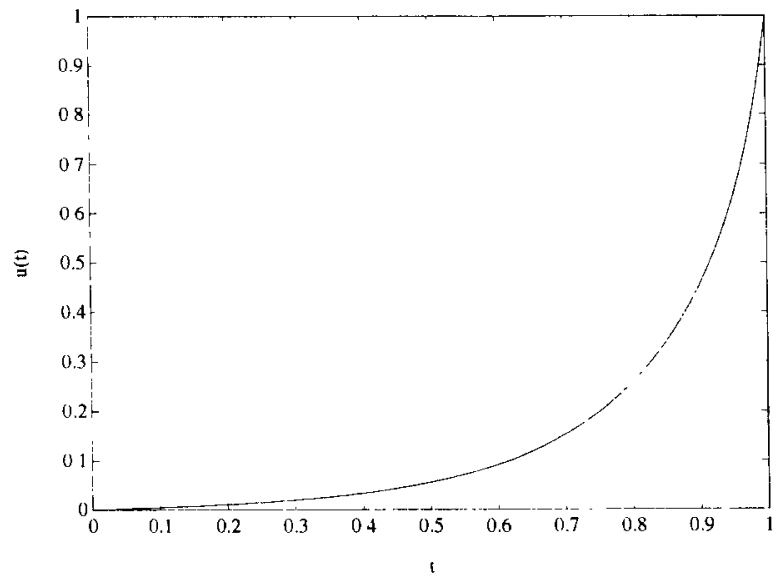

Fig. 4. Solution of problem (32), $\lambda=5$. 
Table 4

Maximum errors for various stepsizes (problem (32))

\begin{tabular}{llllll}
\hline$h$ & \multicolumn{2}{l}{ ETR of order 4} & & \multicolumn{2}{l}{ TOM of order 6 } \\
\cline { 2 - 3 } \cline { 5 - 6 } & $\|e\|_{\infty}$ & rate & & $\|e\|_{\infty}$ & rate \\
\hline 0.1 & $1.8051 \mathrm{e}-1$ & - & $9.0884 \mathrm{e}-2$ & - \\
0.05 & $3.2913 \mathrm{e}-2$ & 2.46 & $1.4653 \mathrm{e}-2$ & 2.63 \\
0.025 & $5.3195 \mathrm{e}-3$ & 2.63 & $1.7345 \mathrm{e}-3$ & 3.08 \\
0.0125 & $6.8539 \mathrm{e}-4$ & 2.96 & $1.3131 \mathrm{e}-4$ & 3.72 \\
0.00625 & $6.9570 \mathrm{e}-5$ & 3.30 & $6.1618 \mathrm{e}-6$ & 4.41 \\
0.003125 & $5.8186 \mathrm{e}-6$ & 3.58 & $1.9127 \mathrm{e}-7$ & 5.01 \\
0.0015625 & $4.2736 \mathrm{e}-7$ & 3.77 & $4.4208 \mathrm{e}-9$ & 5.44 \\
\hline
\end{tabular}

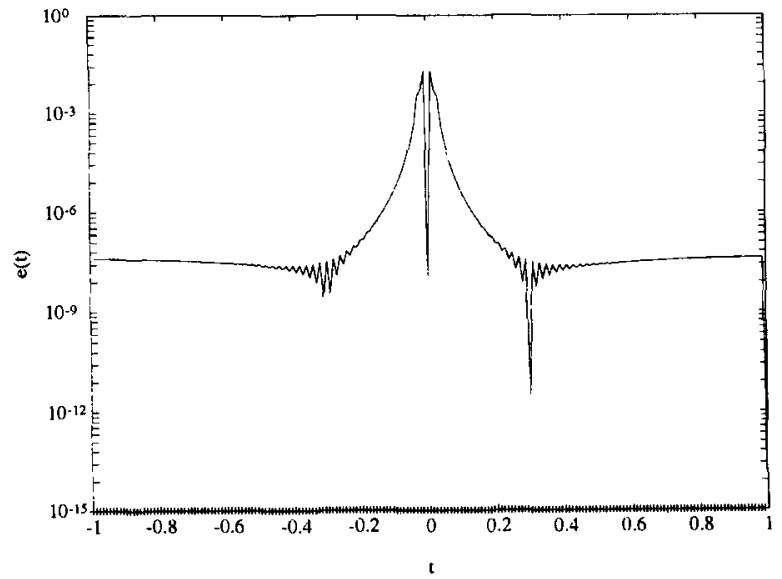

Fig. 5. Error with the ETR of order 4 on problem (31), initial uniform mesh.

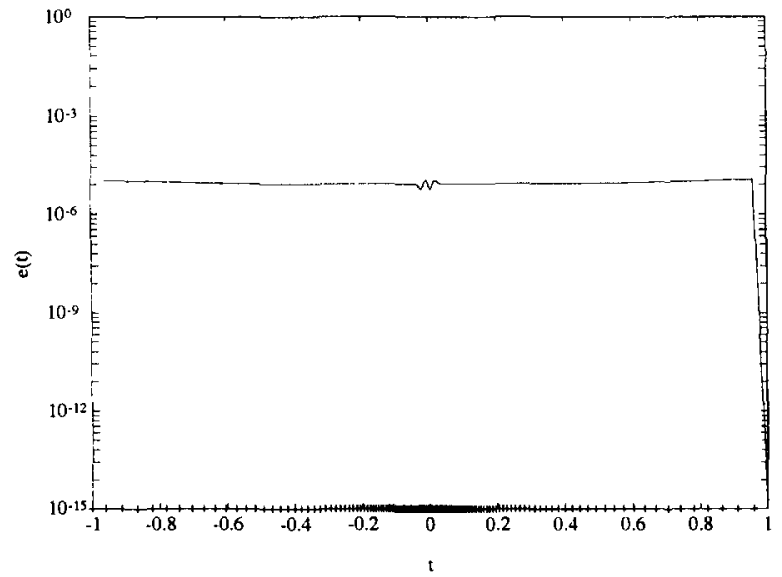

Fig. 6. Error with the ETR of order 4 on problem (31), equidistributed mesh. 


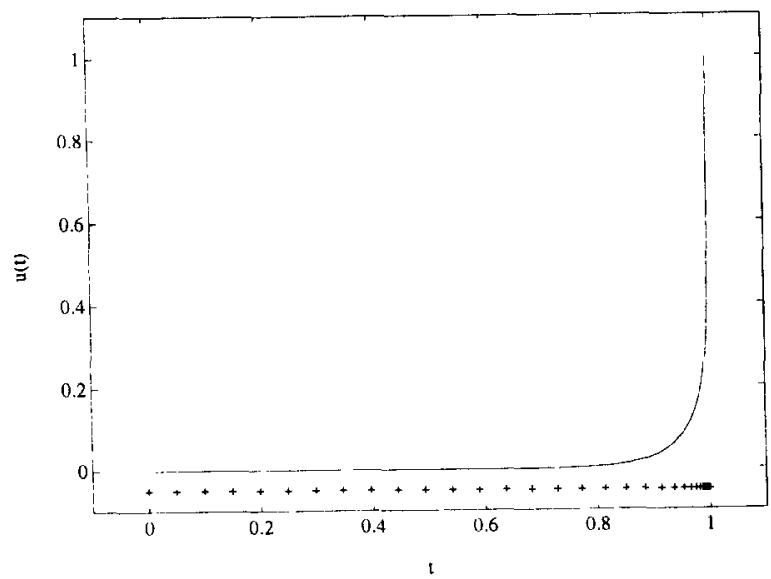

Fig. 7. Computed solution of problem (32), $\lambda=20$, equidistributed mesh.

also shown that the absolute error is now almost uniform (and much smaller), even if the number of the mesh points is the same as in Fig. 5.

This mesh selection technique turns out to be also useful in the nonlinear case. For example, consider the fourth-order ETR applied to solve problem (32) with $\lambda=20$. If a uniform mesh is used, then the intermediate approximations provided by the Newton method highly oscillate near $t=1$ and the method fails to converge. However, if an error equidistribution is made at every intermediate approximation, the Newton method converges smoothly. In Fig. 7 the computed solution is plotted along with the final mesh, which contains 200 points.

\section{Conclusions}

In this paper we have shown how two important classes of BVMs can be used to approximate continuous BVPs. Since these methods can also be used to approximate continuous IVPs, it follows that it is possible to use them both for approximating initial and boundary value problems. In fact the same programs used to carry out the numerical tests can also be used to approximate continuous IVPs.

\section{Acknowledgements}

The authors thank the referee for his comments and suggestions.

\section{References}

[1] P. Amodio, A-stable $k$-step boundary value methods of order $2 k$ for the solution of stiff ODEs (submitted).

[2] P. Amodio, F. Iavernaro and F. Mazzia, Boundary value methods based on Adams-type methods, Rapporto 23/1993, Dipartimento di Matematica dell' Università di Bari, Italy (submitted to the proceedings of NUMD-IFF7). 
[3] P. Amodio and F. Mazzia, A unifying boundary value approach to the numerical solution of ODEs by multistep methods, Rapporto 6/1994, Dipartimento di Matematica dell' Università di Bari, Italy (submitted).

[4] U.M. Ascher, J. Christiansen and R.D. Russel, A collocation solver for mixed order systems of boundary value problems, Math. Comp. 33 (1979) 659-679.

[5] U.M. Ascher and S. Jacobs, On Collocation implementation for singularly perturbed two-point problems, SLAM J. Sci. Statist. Comput. 10 (1989) 533-549.

[6] U.M. Ascher, R.M.M. Mattheij and R.D. Russel, Numerical Solution of Boundary Value Problems for Ordinary Differential Equations (Prentice Hall, Englewood Cliffs, NJ, 1988).

[7] L. Brugnano and D. Trigiante, Stability properties of some BVM methods, Appl. Numer. Math. 13 (1993) 291-304.

[8] L. Brugnano and D. Trigiante, A parallel preconditioning technique for BVM methods, Appl. Numer. Math. 13 (1993) 277-290.

[9] L. Brugnano and D. Trigiante, Convergence and stability of boundary value methods for ordinary differential equations, J. Comput. Appl. Math. (to appear).

[10] L. Brugnano and D. Trigiante, Solving ODE by linear multistep formulae: initial and boundary value methods (in preparation).

[11] P. Marzulli and D. Trigiante, Stability and convergence of boundary value methods for solving ODE, $J$. Difference Equations Appl. 1 (1995) 45-55.

[12] R.M.M. Mattheij, Decoupling and stability of algorithms for boundary value problems, SLAM Rev. 27 (1985) $1-44$.

[13] P. Amodio, V. Golik and F. Mazzia, Variable step boundary value methods based on reverse Adams schemes and their grid distribution, Appl. Numer. Math. (to appear).

[14] J. Stoer and R. Bulirsch, Introduction to Numerical Analysis (Springer-Verlag, Berlin, 1980). 